\title{
Hochwasserschutz für New Orleans - 8 Jahre nach Katrina
}

Im Jahre 2005 verzeichnete New Orleans mit dem Wirbelsturm Katrina eine der folgenreichsten Naturkatastrophen seiner Geschichte, bei der sich auch die Unzulänglichkeit einiger Hochwasserschutzvorkehrungen gezeigt hat. Es waren zahlreiche Tote zu beklagen und die materiellen Schäden waren unübersehbar. Nach dem Ereignis wurde der Hochwasserschutz weiträumig und aufwändig verbessert. Der nachfolgende Beitrag gibt eine Übersicht über den derzeitigen Stand.

\section{Einführung}

Als Nouvelle Orléans im Jahre 1718 gegründet und nach dem Herzog von Orleans benannt wurde, siedelten die französischen Einwanderer auf einem höher gelegenen Gebiet am linken Ufer des Mississippi, dem heutigen französischen Viertel. Die Stadt liegt im Delta des Mississippi (Bild 1), der fast $40 \%$ der zusammenhängenden Fläche der Vereinigten Staaten (ohne Alaska und Hawaii) entwässert und dabei eine große Sedimentfracht mitführt. Diese Sedimente, auf denen die Stadt steht, verdichten sich unter ihrem Eigengewicht, werden (insofern es sich um organische Stoffe handelt) oxydiert oder sinken allmählich in den Golf von Mexiko. Früher konnte dies durch die Sedimentation des mitgeführten Geschiebes und der Schwebstoffe kompensiert werden. Seit der Mississippi eingedeicht und damit bei Hoch- wasser eine Verteilung des Geschiebes über die Fläche des Stadtgebietes unterbunden wurde, sinkt das Gebiet von New Orleans um 5 bis $10 \mathrm{~mm} / \mathrm{a}$, wozu auch das seit etwa 100 Jahren durchgeführte Abpumpen von Drainagewasser und die Erdölförderung (Bild 2) beiträgt [8]. Etwa drei Viertel der Stadtfläche liegen bis zu 1,6 m unterhalb des Meeresspiegels. Dieser Wert würde sich bei einem prognostizierten Meeresspiegelanstieg um $45 \mathrm{~cm}$ bis 2062 noch entsprechend erhöhen. Wenn dieses Szenario eintritt, könnten mittlere jährliche Flutschäden von 23 Mrd. US-Dollar zu erwarten sein.

New Orleans ist von zwei Seiten hochwassergefährdet: Zum einen durch den Mississippi und zum anderen durch Wellen sowie Sturmfluten vom Meer. Die Stadt ist von einigen lagunenartigen Seen umgeben, die mit dem Golf von Mexiko verbunden sind und deren größter der
Pontchartrain See im Norden der Stadt mit einer Fläche von $1839 \mathrm{~km}^{2}$ (ca. dreimal so groß wie die Fläche des Bodensees), mit einer Ost-West-Ausdehnung von etwa $64 \mathrm{~km}$ und mit einer durchschnittlichen Tiefe von etwa $4 \mathrm{~m}$ ist. Im Bereich von Schifffahrtsrouten wurde er teilweise künstlich vertieft.

Entlang des eingedeichten Mississippi erstreckt sich beidseitig flussaufwärts von New Orleans auf einer Länge von etwa $90 \mathrm{~km}$ bis nach Baton Rouge der Hafen von Süd-Louisiana als Umschlagstelle zwischen Binnen- und Seeschifffahrt (Bild 3). Im Stadtzentrum an der Kanalstraße ist der Mississippi ca. $700 \mathrm{~m}$ breit und stellenweise bis zu $50 \mathrm{~m}$ (Flussmitte) bzw. $18 \mathrm{~m}$ (in Ufernähe) tief. Der Flusswasserspiegel liegt hier noch deutlich über dem Meeresspiegel, weshalb die den Fluss kreuzenden Wasserstraßen durch Schiffsschleusen angebunden sind.

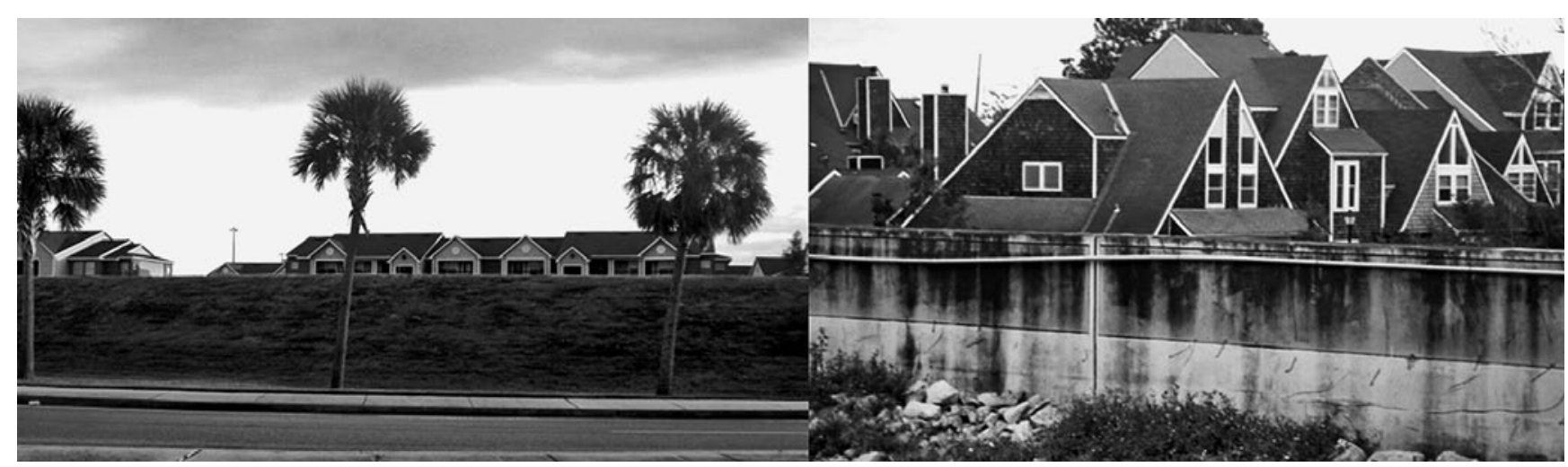

Bild 1: Hochwasserschutzanlagen im Norden von New Orleans am Pontchartrain See: links: Deich an der Uferstraße (Lakeshore Drive); rechts: Hochwasserschutzwand und direkt dahinter stehende Häuser am Ablaufkanal an der 17. Straße (In beiden Fällen ist die Sichtbeziehung der Wohnhäuser zum Wasser gestört und besteht nur noch in der oberen Etage; vermutlich aus Kostengründen wurde dennoch auf Glaswände verzichtet) 


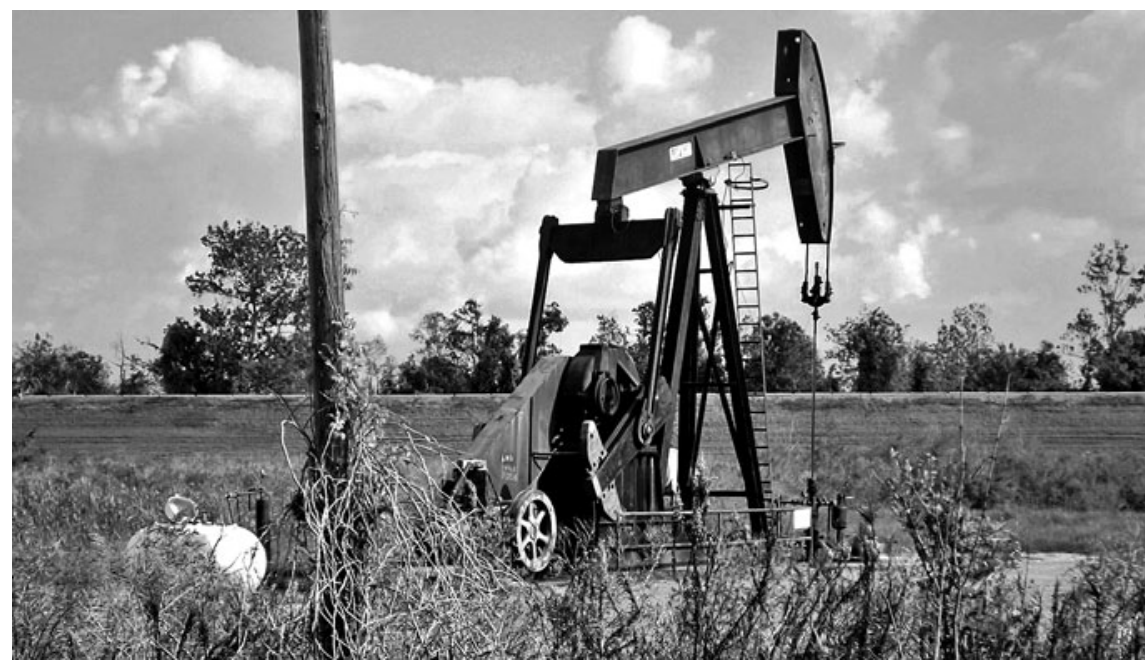

Bild 2: Erdölförderung durch eine große US-Mineralölgesellschaft direkt hinter dem Deich mit daneben befindlicher Raffinerie (nicht im Bild), die Erdölförderung trägt auch zur Geländeabsenkung bei

\section{Hochwasserereignisse und Folgen}

In der Vergangenheit ist New Orleans schon oft von Hochwasserereignissen betroffen gewesen. Wegen des geringen Längsgefälles von nur $0,00022=0,22 \%$ o im Mittellauf bis zur Ohio-Mündung bei Cairo und des noch geringeren Gefälles von nur $0,00006=0,06 \%$ auf den letzten $1600 \mathrm{~km}$ ist das Gebiet beidseits des Mississippi hochwassergefährdet und wäre ohne Hochwasserschutzanlagen häufigen Überflutungen ausgesetzt.
Das Hochwasser des Mississippi im Jahre 1927 gilt als eine der größten Naturkatastrophen in der Geschichte der USA. 250 Tote waren damals zu beklagen. Ein weiteres Extremhochwasser im Frühjahr 1973 forderte 33 Todesopfer.

Als nach einem schneereichen Winter Ende April 2011 starke Niederschläge im Mississippi-Einzugsgebiet fielen, kam es im Mai 2011 wiederum zu einem bedeutenden Hochwasser. Für den 18.3.2011 wurde für Vicksburg, wo sich die Wasserstraßen-Versuchsstation (W.E.S.) und das Ingenieur-, Forschungs- und Entwick-

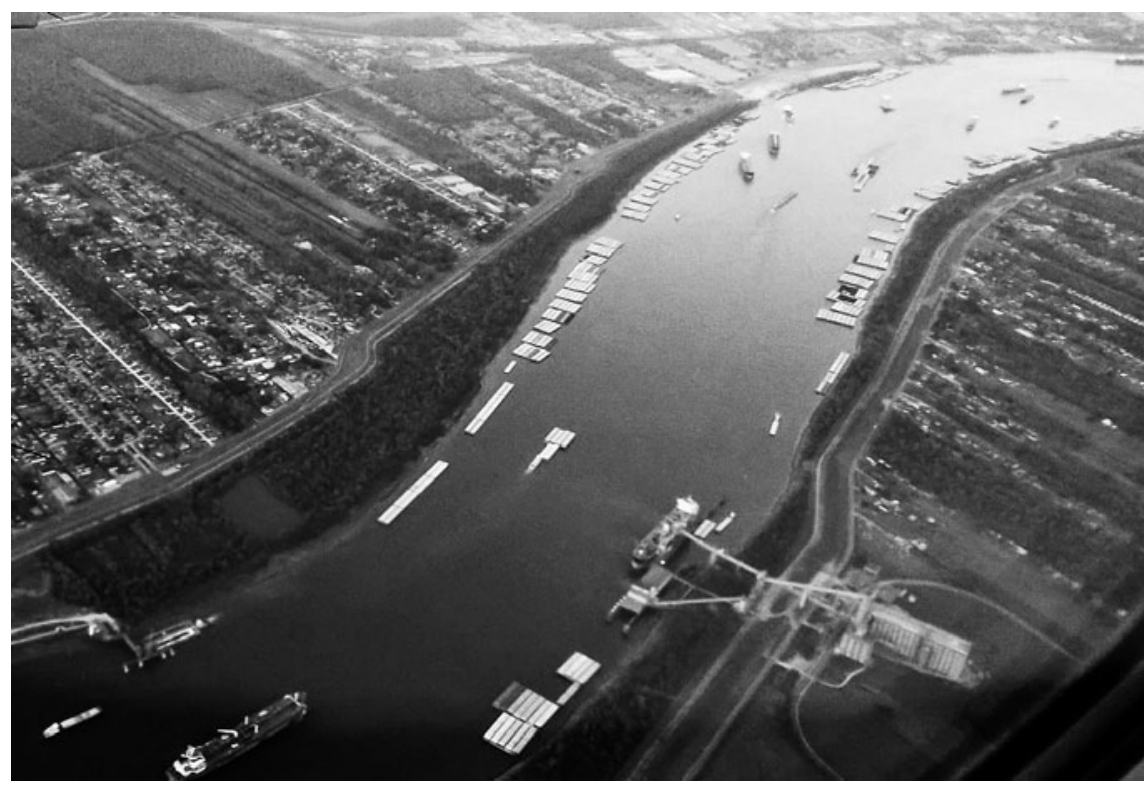

Bild 3: Hafen von Süd-Louisiana, der sich auf ca. $90 \mathrm{~km}$ zwischen Baton Rouge und New Orleans an beiden Ufern des Mississippi erstreckt (Blick in Fließrichtung), gut sichtbar sind die beidseitigen Hochwasserschutzdeiche sowie die Seeschiffe und die Binnenschiffe (Schubverbände mit Leichtern) lungszentrum (E.R.D.C.) des Ingenieurkorps der US-Streitkräfte (USACE) befindet, ein Durchfluss von $66000 \mathrm{~m}^{3} / \mathrm{s} \mathrm{ermit-}$ telt. Bei diesem Ereignis wurde durch die zuvor ertüchtigten Hochwasserschutzanlagen und eine optimale Steuerung von Entlastungsanlagen ein sehr viel größerer Schaden verhindert [1].

In der Stadtgeschichte von New Orleans haben schon einige Wirbelstürme, die seit den fünfziger Jahren des 20. Jahrhunderts mit Vornamen bezeichnet wurden, die Bewohner heimgesucht, so z. B. 1856, 1860, 1879, 1893, 1909, 1915, 1940, 1947, 1957 (Audrey), 1965 (Betsy), 1969 (Camille) und 1992 (Andrew). Schon vor 2005 war die Stadt bereits fünfmal überflutet worden: 1915, 1940, 1947, 1965, 1969.

Eines der größten und folgenreichsten, von der Seeseite verursachten Hochwasser seit der Stadtgründung war der Wirbelsturm (Hurrikan) Katrina vom 23. bis 30.8.2005, der die Bundesstaaten Florida, Louisiana (besonders den Großraum New Orleans), Mississippi, Alabama und Georgia betraf. Im Südosten der USA, insbesondere am Golf von Mexiko, wurde dem Ereignis zeitweise die höchste Stufe 5 auf der Saffir-Simpson-Skala zugeordnet. Das Minutenmittel der Windgeschwindigkeit betrug in der Spitze $280 \mathrm{~km} / \mathrm{h}$ und die Niederschlagshöhe in New Orleans erreichte $250 \mathrm{~mm}$, örtlich bis $380 \mathrm{~mm}$ in 24 Stunden. Die Höhe der Sturmflut betrug örtlich bis zu $8 \mathrm{~m}$ und die Höhe der Windwellen $16 \mathrm{~m}$, wobei allerdings die zugehörige Überschreitungswahrscheinlichkeit aus dem Spektrum nicht genau angegeben wird [2].

Nach entsprechenden Warnungen verließen mehr als 1 Mio. Menschen die Stadt und die Gegend um New Orleans in Richtung des nördlichen Louisiana und gelangten in Privatfahrzeugen und Bussen teilweise bis nach Texas. Dies war der größte Exodus seit dem nordamerikanischen Bürgerkrieg (1861 bis 1865). Viele von ihnen sind bis heute nicht zurückgekehrt (Bild 4) und z. B. in Baton Rouge geblieben, was dem Vernehmen nach die dortige Infrastruktur dauerhaft überfordert. Mit dem Wegzug vieler sozial schwächer gestellter Bewohner und dem sich in jüngster Zeit wieder verstärkenden Zuzug als Folge einer wirtschaftlichen Wiederbelebung hat sich auch die Sozialstruktur in Teilen von New Orleans und seiner Nachbargemeinden verändert.

Während die Flussdeiche entlang des Mississippi hielten, führten in New Orle- 


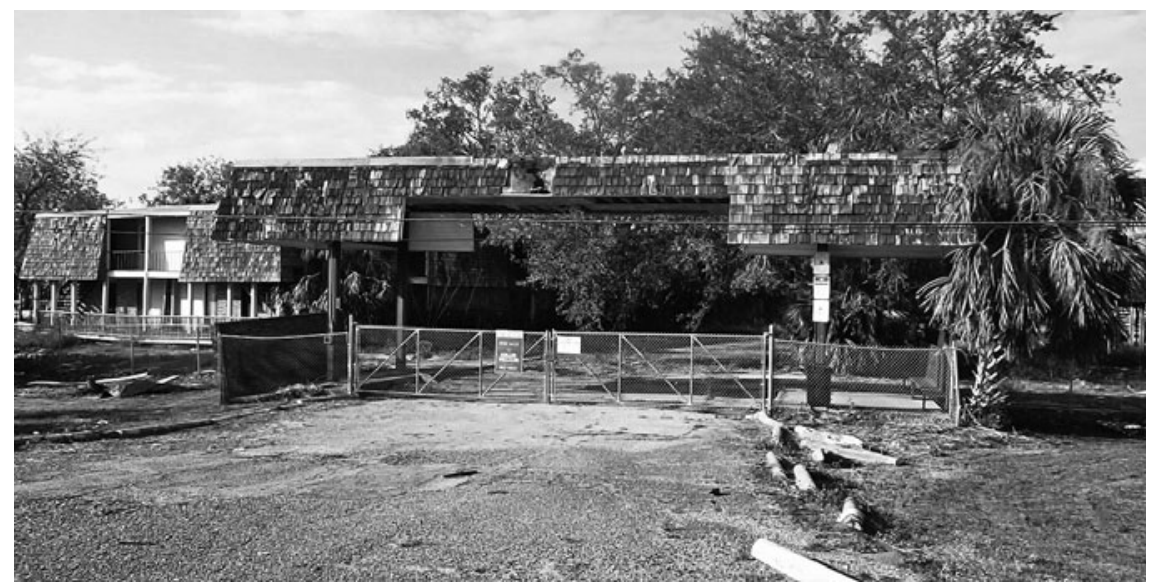

Bild 4: Aufgegebene Siedlungen im Osten von New Orleans (Village de l'est)

ans mehrere Brüche im Deichsystem insbesondere an Kanälen zum Pontchartrain See, wie z. B. in den Ablaufkanälen an der 17. Straße und der London Avenue, dazu, dass ca. $80 \%$ des Stadtgebietes bis $\mathrm{zu}$ 7,60 m tief unter Wasser standen. Nur einige höher gelegene Bereiche, wie das französische Viertel, wurden nicht überflutet. Durch Gründungsversagen ereignete sich z. B. auch ein Bruch an der nördlichen Hochwasserschutzwand auf der Ostseite des Binnenhafen-Schifffahrtskanals (Inner Harbour Navigation Canal, IHNC). Im Großraum New Orleans gab es insgesamt ca. 50 Deichbrüche [8]. 34 der 71 Schöpfwerke in der Stadt wurden beschädigt oder konnten aus anderen Gründen nicht in Betrieb gehen. $270 \mathrm{~km}$ der $560 \mathrm{~km}$ langen Hochwasserschutzanlagen des Gesamtsystems der Region waren in Mitleidenschaft gezogen worden.

Die Breschen konnten erst eine Woche später vollständig geschlossen werden. Mehrere Zehntausend Menschen, die die Stadt nicht verlassen konnten, suchten $\mathrm{Zu}$ flucht im Louisiana Superdome (jetzt Mercedes-Benz-Superdome, Bild 5), der aber dann auch vom Wasser eingeschlossen war und evakuiert werden musste. Mehrere Brücken einschließlich der Verbindung über die Interstate 10 nach Slidell wurden zerstört, auch die Straßenanbindung über Land war unterbrochen und für 900000 Menschen fiel die Elektroenergieversorgung aus, weshalb auch diejenigen Schöpfwerke ausfielen, die nicht über eine Notstromversorgung verfügten. Einer von zwei Flughäfen war überflutet und musste seinen Betrieb einstellen. Auf dem zweiten Flughafen mussten bis zu 300 Bewegungen täglich ohne Radar und Bodenbeleuchtung abgewickelt werden. Wegen des Ausfalls der Trinkwasserversorgung wa- ren die hygienischen Zustände in der Stadt bedenklich. Das Oberflächenwasser, mit dem die Menschen in Berührung kamen, war mit Öl, Benzin, Chemikalien und Fäkalien (hoher Colititer) verunreinigt und deren Erkrankungen, die vor allem auch in den Auffanglagern festgestellt wurden. Die Nationalgarde musste eingesetzt werden, um Plünderungen und andere kriminelle Aktivitäten einzudämmen.

Die traurige Bilanz des Naturereignisses war der Verlust von rund 1800 Menschenleben. Viele, vor allem Ältere und Behinderte ertranken, weil sie dem nach den Deichbrüchen mit $30 \mathrm{~cm}$ pro $10 \mathrm{Mi}$ nuten ansteigenden Wasserspiegel nicht entfliehen konnten [8]. Es entstand ein Sachschaden, der entsprechend dem Preisindex von 2005 auf 108 Mrd. US-Dollar beziffert wurde.

Das für den Hochwasserschutz am Mississippi und in New Orleans verantführte zu Magen-, Darm-, Haut- und an- wortliche Ingenieurkorps der US-amerikanischen Streitkräfte (US-Army Corps of Engineers, USACE) gründete unmittelbar nach dem Hurrikan Katrina eine Einsatzgruppe zur Reparatur und Wiederherstellung der Hochwasserschutzanlagen, die spätestens zum Beginn der Hurrikan-Saison des Folgejahres wieder funktionsfähig sein mussten. Eine zweite Einsatzgruppe war damit beschäftigt, ca. $1 \mathrm{Mrd} . \mathrm{m}^{3}$ Wasser in 53 Tagen abzupumpen [2].

\section{Anpassung des Hochwasser- schutzes nach Katrina}

Die Stadt muss sowohl gegen die Hochwasserereignisse des Mississippi als auch gegen Meereshochwasserereignisse und Seegang geschützt werden.

Für den Schutz vor den durch den Mississippi herangeführten Hochwasserabflüssen stehen im südlichen Louisiana drei bedeutende Entlastungsmöglichkeiten zur Verfügung: zwei in der Nähe von Morganza (ein wasserstandsabhängiger freier seitlicher Überfall und ein rechtsseitiges Entlastungswehr) nördlich von Baton Rouge und eine direkt oberstrom des eingedeichten Stadtgebietes von New Orleans. Die beiden Erstgenannten ermöglichen den Abschlag von jeweils ungefähr 17000 m³ in das Flussgebiet des Atchafalaya, der westlich in den Golf von Mexiko mündet. Die dritte Entlastung, das Bonnet-CarréGerinne, leitet das Hochwasser westlich von New Orleans in den Pontchartrain-See (Bild 6). Auf diese Weise wird das höherwertige Stadtgebiet von New Orleans geschützt, wobei in den überfluteten Berei-

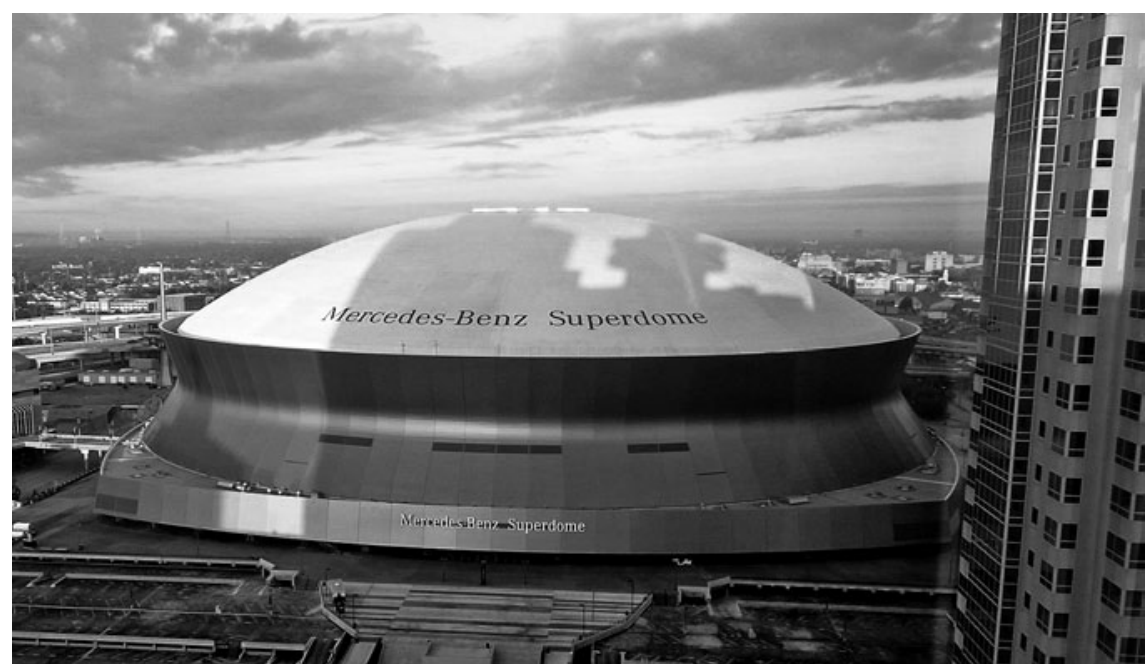

Bild 5: Während des Hurrikans Katrina war der Superdome Zufluchtsstätte für viele Einwohner 


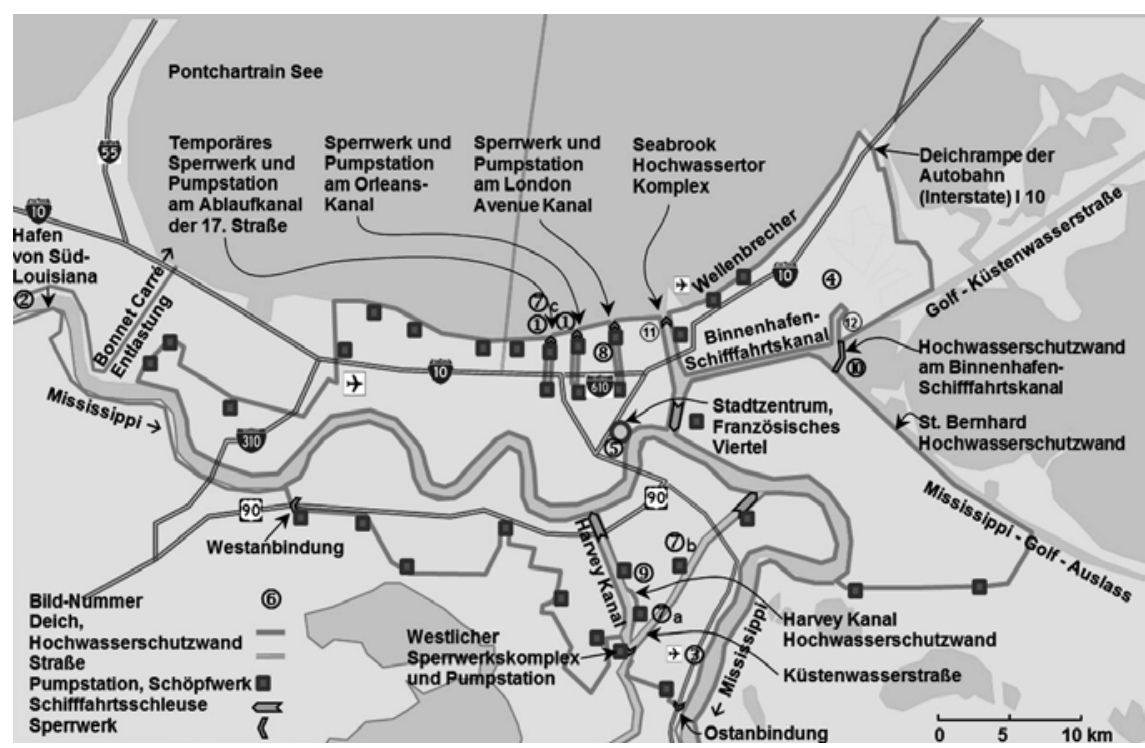

Bild 6: Übersicht über die wichtigsten Hochwasserschutzanlagen von New Orleans

chen des Atchafalaya-Einzugsgebietes bewusst Schäden in Kauf genommen werden.

Während des Hochwassers 2011 wurden erstmals alle drei Entlastungen in Anspruch genommen, um New Orleans vor dem Hochwasser zu schützen. Das Entlastungswehr bei Morganza war zu 1/5 geöffnet.

Da im Gebiet um New Orleans das hochliegende Gelände fehlt, an welches Hochwasserschutzanlagen üblicherweise anschließen, bleibt nur die Möglichkeit,
Ringdeiche zu errichten, die auf der einen Seite Flussdeiche oder Hochwasserschutzwände entlang des Mississippi sowie verschiedener Kanäle und auf der anderen Seite Seedeiche mit Sperrwerken sind. Die großräumig angelegten Ringdeiche links (nördlich) und rechts (südlich) des Mississippi sind nach 2005 auf das 100-jährliche Ereignis als Schutzziel ausgelegt worden (Bild 1). Wohl wissend dass dies kein vollständiger Schutz sein kann, wird vom
USACE auch deutlich kommuniziert, dass näherungsweise in einem von 100 beliebigen Jahren das Bemessungsereignis überschritten wird. Deshalb wird auch nicht von einem Hochwasserschutzsystem gesprochen, sondern von einem „System zur Verminderung des Schadensrisikos bei Wirbelstürmen und Unwettern" (Hurricane and Storm Damage Risk Reduction System; HSDRRS).

Nach dem Wirbelsturm Katrina im Jahre 2005 wurde dieses System wiederhergestellt und an vielen Stellen verbessert. Insgesamt gab es 59 Projekte, die von 26 Bauunternehmen realisiert wurden. Es wurden Deiche und Hochwasserschutzwände erneuert und erhöht, wobei die Hochwasserschutzlinie der Stadt um $87 \mathrm{~km}$ auf $352 \mathrm{~km}$ verkürzt wurde [2]. Von den 78 Pumpwerken wurden viele seit 2005 repariert. Die Pumpwerke an den drei großen nördlichen Ablaufkanälen (17. Straße, London Straße, Orleans Straße) wurden neu errichtet (Bild 7).

Die Hochwasserschutzwände als Deichverlängerung und zur Deicherhöhung wurden häufig als I-Typ (z. B. Betonwand als Kopfbalken auf Spundwand zur Deichoder Untergrundabdichtung; Bild 8) oder T-Typ = Winkelstützmauer (Winkelstützmauer in Form eines auf dem Kopf stehenden T; Bild 9) errichtet.

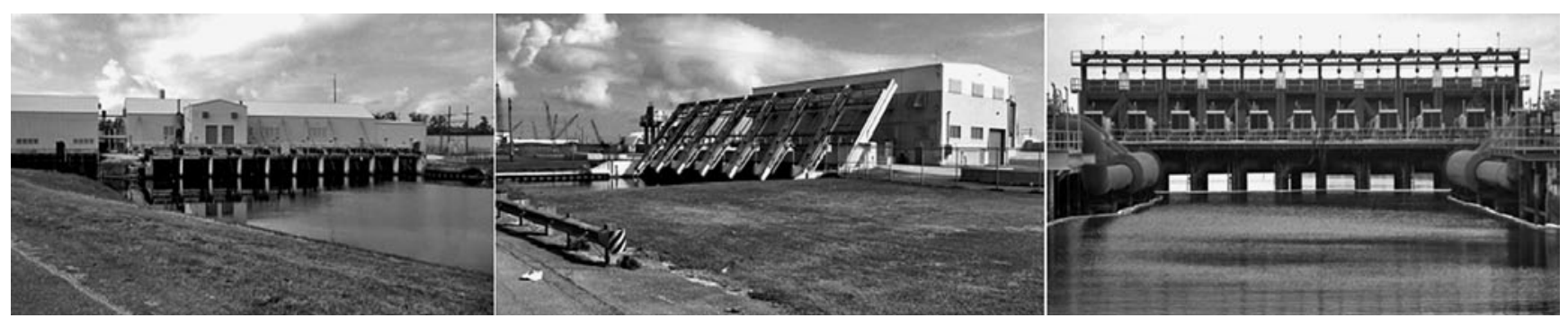

Bild 7: Pumpstationen in New Orleans: Harvey Kanal; Engineers Road am Intracoastal Waterway; Temporäres Hochwassersperrwerk mit Pumpstation, New Orleans Ablaufkanal an der 17 Straße am Pontchartrain See im Norden der Stadt (v. I. n. r.)

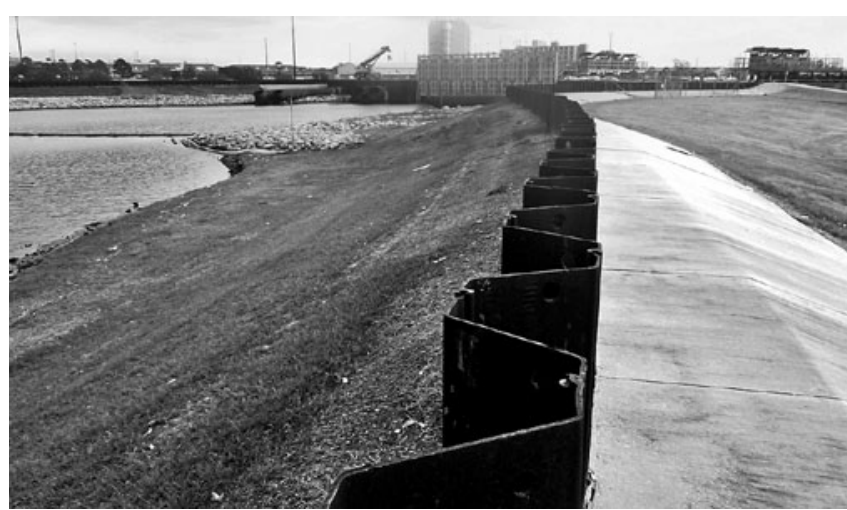

Bild 8: Deicherhöhung mittels Spundwand und luftseitigem Betonwiderlager am London Avenue Kanal, New Orleans

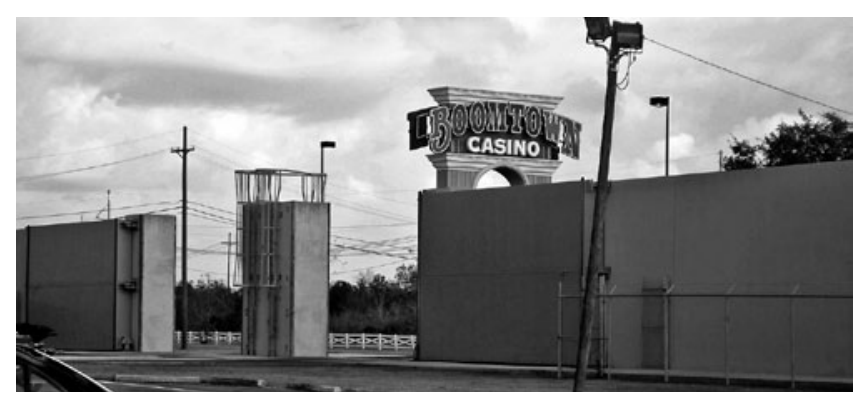

Bild 9: Neu errichtete Hochwasserschutzwand am Harvey Kanal (Harvey Canal Floodwall) mit Toren und Übersteigmöglichkeit in New Orleans (Das „Boomtown“ Casino befindet sich auf der Wasserseite und wird nur durch den alten, weniger hohen Deich geschützt, den man jetzt als Teilschutzdeich bezeichnen kann.) 


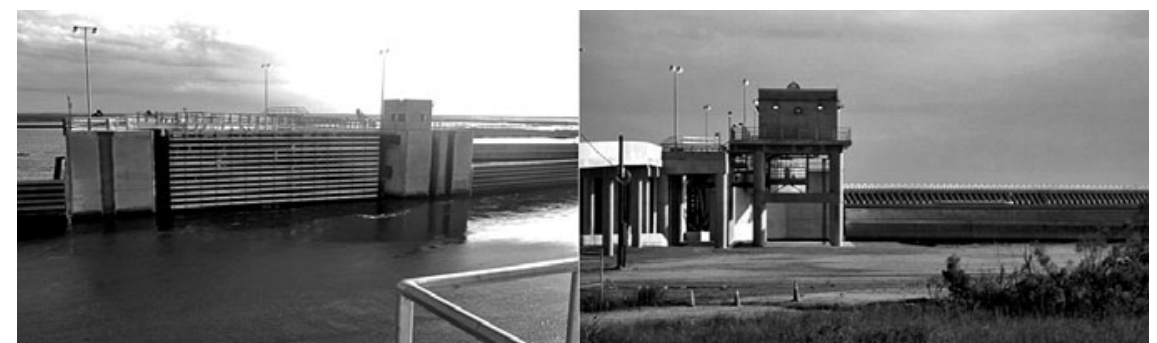

Bild 10: Sperrwerk am Binnenhafen-Schifffahrtskanal in New Orleans (IHNC Surge Barrier Wall): links: mit Stemmtoren verschließbare Schiffsdurchfahrt; rechts: nach Süden anschließende Pfeilerstauwand

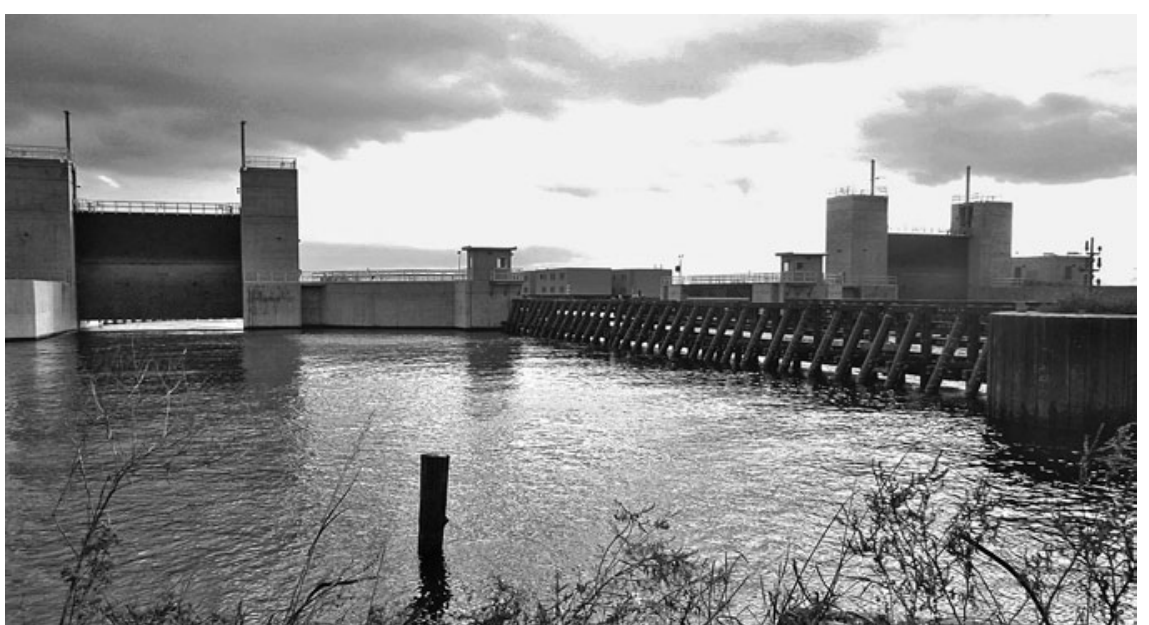

Bild 11: New Orleans Seabrook Hochwassertore im Norden der Stadt am Pontchartrain See (Hubtore und Stemmtor)

Ein Drainagesystem mit Ablaufkanälen (Outfall Canals) von mehreren hundert Kilometern Länge durchzieht heute die Stadt und entwässert sie über 78 Pumpstationen. Durch die weiträumige Trockenlegung konnte die Fläche von New Orleans deutlich erweitert werden. Andererseits wird dadurch auch die Geländesenkung begünstigt. Die Stadt ist heute im Norden von einem $5 \mathrm{~m}$ bis $6 \mathrm{~m}$ hohen Deich und im Süden von einem bis zu $9 \mathrm{~m}$ hohen Deich gegen den plötzlichen Wassereintritt geschützt. Der Sickerwasserzutritt kann allerdings naturgemäß nicht vollständig unterbunden werden.

Die Wiederherstellung und Erweiterung des HSDRRS wurde auf Beschluss des Kongresses von der Bundesregierung in der Größenordnung von 14 Mrd. USDollar finanziert [4] und ist unter der Leitung sowie Koordination des USACE in Zusammenarbeit mit nationalen, bundesstaatlichen und lokalen Partnern bis heute nahezu fertiggestellt. Der von 2008 bis 2011 vorgesehene Teilbetrag von 5,7 Mrd. US-Dollar entsprach ungefähr dem Betrag, der sonst dem USACE auf gesamtnationaler Ebene zur Verfügung stand. liche Sperrwerkskomplex (West Closure Complex, WCC). Das IHNC-Sperrwerk ist weltweit das größte seiner Art und weist konstruktive Gemeinsamkeiten mit einer Pfeilerstaumauer auf (Bild 10). Es schützt die Stadt vor dem Hochwasser aus östlicher Richtung, wo sich der BorgneSee befindet. Das Sperrwerk verfügt über einen schiffbaren Durchlass des Golf-Küsten-Wasserweges (Gulf Intrcoastal Waterway, GIWW), der mit großen Stemmtoren ausgerüstet ist und über einen weiteren Durchlass für den Mississippi-Golf-Auslass (Mississippi River Gulf Outlet), welcher entlang der St.-Bernard-Hochwasserschutzwand verläuft. Der Seabrook-Hochwassertor-Komplex bildet den Abschluss des IHNC nach Norden zum Pontchartrain See und wurde für Baukosten von ca. 165 Mio. US-Dollar errichtet [4]. Das zweiteilige Stemmtor und beidseits ein Hubtor sind im Normalbetrieb für den Schiffsund Bootsverkehr geöffnet und werden bei Hochwasser geschlossen (Bild 11). Das weltgrößte Pumpwerk [2] befindet sich südlich des Stadtzentrums an der Vereinigung des Harvey-Kanals mit der Küstenwasserstraße (Intracoastal Waterway). Durch die Tore des westlichen Sperrwerks WCC ist die Durchgängigkeit für den Schiffsverkehr gegeben. Als Gesamtbaukosten für diesen Komplex aus Sperrwerk, Durchlass und Pumpwerk wird etwa $1 \mathrm{Mrd}$. US-Dollar angegeben [4].

Öffnungen und Durchlässe sind aber nicht nur für Wasserwege, sondern auch für Straßen und Schienen notwendig (Bild 9). Ein Beispiel hierfür ist die Ostanbindung

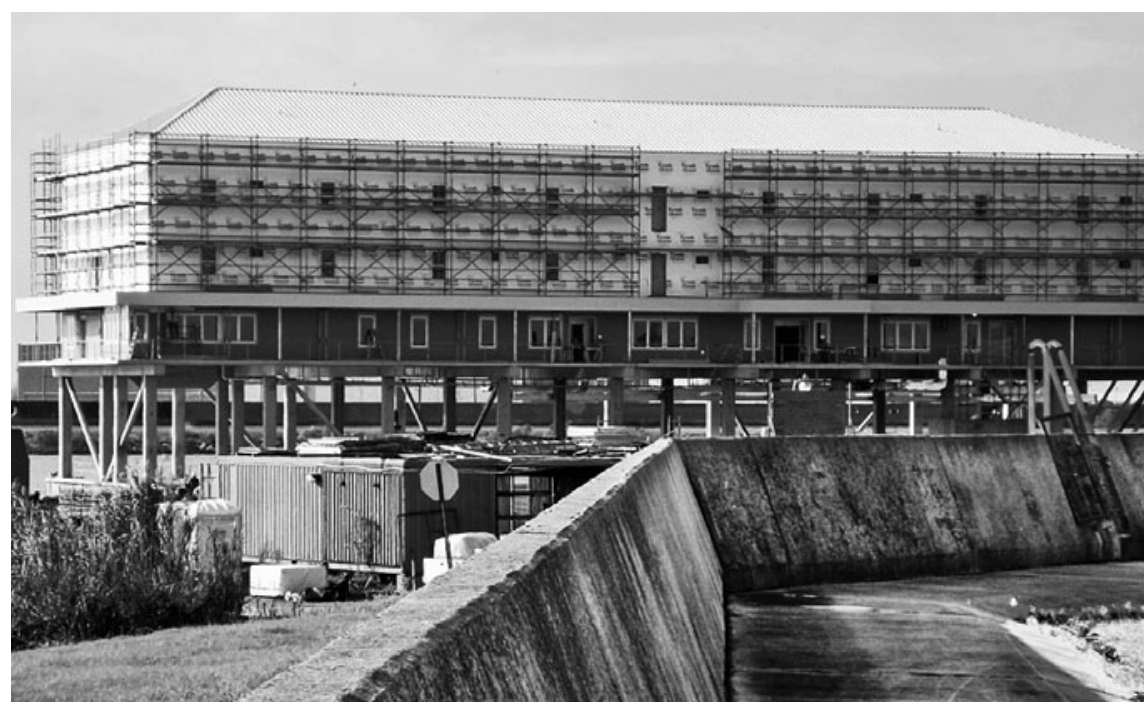

Bild 12: Hochwasserangepasstes Bauen in New Orleans am Industrial Parkway: im Vordergrund mit einer Hochwasserschutzwand (T-Typ) erhöhter Deich; das auf Pfählen errichtete Bürogebäude steht wasserseitig der Hochwasserschutzlinie 
(Eastern Tie-In). Die Staatsstraße Nr. 23 und die parallel verlaufende Eisenbahnlinie werden durch Deichscharten geführt, die durch große Stahltore mit senkrechter Drehachse verschlossen werden können. In der Straßenmitte gibt es dort für das zweiflüglige Tor einen versenkbaren Anschlagpfosten.

An anderen Stellen wird die Hochwasserschutzlinie über Deichrampen oder Brücken gekreuzt. Ein Beispiel hierfür ist die Autobahn Interstate 10, die die Stadt am nordöstlichen Rand in Richtung Slidell verlässt und dabei den Hochwasserschutzdeich kreuzt.

Im Sommer 2012 hat das Ingenieurkorps der US-amerikanischen Streitkräfte die Hochwasserschutzanlagen von New Orleans einer Überprüfung mit Funktionskontrolle unterzogen. Der Testlauf für die Deiche, Wände, Tore und Pumpen war erfolgreich und kam zur rechten Zeit, denn der Tropensturm „Debby“ war im Anzug und sollte anderenorts zu Schäden führen [4].

In Ergänzung zu den öffentlichen Schutzmaßnahmen wird auch individuelle Vorsorge betrieben. Beispiele hierfür sind lokale ringförmige Hochwasserschutzlösungen außerhalb des geschützten Gebietes sowie Pfahlbauten innerhalb und außerhalb der Deichlinie (Bild 12).
Zusätzlich zu den technischen Maßnahmen wurde versucht, das Ökosystem der Feuchtgebiete außerhalb der Deichlinie zu schützen und wenn möglich und erforderlich wieder herzustellen, wofür ein gesonderter Plan entwickelt wurde.

\section{Schlussfolgerungen und Ausblick}

Der US-Bundesstaat Louisiana an der Mündung des Mississippi in den Golf von Mexiko wird häufig von Hochwasserereignissen des Mississippi und Hurrikans mit Sturmfluten von See heimgesucht. Nach der Flutkatastrophe im Zuge des Hurrikans Katrina im Jahre 2005 wurde der damals unzureichende Hochwasserschutz mit enormem finanziellem Aufwand wesentlich verbessert und auf das 100 -jährliche Ereignis ausgerichtet. Eine erste Bewährungsprobe hat das flussseitige Hochwasserschutzsystem beim Mississippi-Hochwasser 2011 bestanden.

Da die zusammenhängenden geschützten Flächen teilweise sehr groß und zahlreiche Verschlüsse vorhanden sind, könnten gegebenenfalls Kammerungen eine noch größere Hochwassersicherheit ermöglichen, falls einzelne Abschnitte der Hochwasserschutzlinie versagen sollten.

\section{Reinhard Pohl}

\section{Flood Protection for New Orleans - 8 Years after Katrina}

In 2005 the City of New Orleans had to suffer one of the most momentous natural disasters in its history during which also the insufficiency of some flood protection measures became visible. Many people were killed and the material damage was immense. After this event the flood protection has been improved wide ranging and very costly. This paper gives an overview.

\section{Рейнхард Поль}

\section{Противопаводковая защита Нового Орлеана - спустя 8 лет после урагана «Катрина»}

В 2005 году в Новом Орлеане прошел ураган «Катрина» - один из самых крупных природных катаклизмов в истории города. В этот период проявилась явная недостаточность некоторых мер противопаводковый защита. Ураган повлек за собой многочисленные жертвы и огромные материальные потери. После такой катастрофы система противопаводковой защиты была улучшена в значительном объеме, что потребовало немалых затрат. Данная статья представляет собой обзор состояния в настоящий момент.

Weil die Abgrenzung der Hochwasserschutzanlagen in unterschiedlichen Quellen abweichend vorgenommen wird, differieren die Angaben über Längen und Kosten teilweise.

Infolge der Senkung des Stadtgebietes und des Anstieges des Meeresspiegels wird es auch weiterhin Anpassungsbedarf für die Hochwasserschutzanlagen geben, um New Orléans das Schicksal der sagenumwobenen Stadt Vineta zu ersparen. Die Zukunft wird zeigen, wie lange und in welchem Umfang ein Hochwasserschutz wirtschaftlich sowie technisch möglich sein wird und den Lebensraum der Menschen in der gerade wieder aufstrebenden, wachsenden Metropolregion New Orleans dauerhaft $\mathrm{zu}$ sichern vermag.

Autor

Prof. Dr.-Ing. habil. Reinhard Pohl

Institut für Wasserbau und

Technische Hydromechanik der TU Dresden 01062 Dresden

Reinhard.Pohl@tu-dresden.de

\section{Literatur}

[1] Kron, W., Steuer, M.: Mississippi-Flut 2011. In: Korrespondenz Wasserwirtschaft 5 (2012), Nr. 5, S. 245-250.

[2] US Army Corps of Engineers: New Orleans District (www.mvn.usace.army.mil, Aufruf: 20.05.2013).

[3] U.S. Geological Survey (Hrsg.): Geomorphic Changes on the Mississippi River Flood Plain at Miller City Il. as a Result of the Flood of 1993. U.S. Geological Survey Circular 1120-J.

[4] N. N.: Sieben Jahre nach Katrina, Big Easy ist schon wieder ziemlich big. In: FAZ, 3.7.2012.

[5] N. N.: Fakten und Zahlen zum Hochwasserschutz in Louisiana. In: Die Presse, 29.08.2012.

[6] Bea, R. G.: Failure of the New Orleans $17^{\text {th }}$ Street Canal Levee and Floodwall during Hurricane Katrina. In: Proc. GeoCongress 2008, New Orleans, LA. 9.-12.3.2008.

[7] Noack, T.: DerHurrikan Katrina und seine Auswirkungen auf New Orleans, USA. Abschlussarbeit im Rahmen der Ersten Staatsprüfung für das Lehramt, Christian-AlbrechtsUniversität zu Kiel, Geographisches Institut, 2007.

[8] Daniel, D. E. et. al.: The New Orleans Hurricane Protection System: What went wrong and why? In: American Society of Civil Engineers (Hrsg.): Report by the American Society of Civil Engineers Hurricane Katrina External Review Panel, Reston, Virginia, 2007.

[9] Sills, G. L.; Vroman, N. D.; Wahl, R. E.; Schwanz, N. T.: Overview of New Orleans Levee Failures: Lessons Learnedand Their Impact on National Levee Design and Assessment. In: Journal of Geotechnical and Geoenvironmental Engineering (2008), S. 556-565. 